\title{
Development of an FVIII Inhibitor in a Mild Hemophilia Patient with a Phe595Cys Mutation
}

\author{
Jun Yamanouchi ${ }^{1}$, Daiki Tokumoto ${ }^{1}$, Yuichi Ikeda ${ }^{1}$, Masaki Maruta ${ }^{1}$, Masahiko Kaneko ${ }^{2}$, \\ Takaaki Hato $^{3}$ and Masaki Yasukawa ${ }^{1}$
}

\begin{abstract}
:
Mild hemophilia A is caused by a missense mutation in the FVIII gene that is responsible for a decrease in the FVIII:C to between 5\% and 40\%. The development of FVIII inhibitors has been reported in 3-13\% of patients with mild hemophilia. Genetic risk factors for the development of inhibitors in mild hemophilia have been investigated. In the present study, we encountered a case of mild hemophilia with an FVIII inhibitor and identified the mutation responsible: a novel Phe595Cys mutation in the FVIII gene. In addition, this study showed that the inhibitor recognized exogenous wild-type FVIII and autologous mutant FVIII.
\end{abstract}

Key words: FVIII inhibitor, mild hemophilia A, neutralization

(Intern Med 57: 3179-3182, 2018)

(DOI: 10.2169/internalmedicine.1145-18)

\section{Introduction}

Mild hemophilia A is commonly caused by a missense mutation in the factor VIII (FVIII) gene, which results in a decrease in the FVIII:C to between 5 and $40 \mathrm{U} / \mathrm{dL}$ (1). Patients with mild hemophilia may develop inhibitors, even though the incidence of inhibitor development is markedly lower than that in patients with severe hemophilia. The incidence of inhibitor development in patients with mild hemophilia has been reported to be between $3 \%$ and $13 \%(2,3)$.

Risk factors for the development of inhibitors in hemophilia are a family history of inhibitors, intensive FVIII treatments, old age at the first exposure, a high number of peak treatments, and certain missense mutations (3).

Genetic risk factors for the development of inhibitors in patients with mild hemophilia have been examined, and the Arg593Cys and Trp2229Cys mutations in FVIII were identified $(4,5)$. We herein report a mild hemophilia patient carrying the Phe595Cys mutation who developed an inhibitor that neutralized exogenous wild-type FVIII and autologous mutant FVIII.

\section{Case Report}

A 68-year-old man was diagnosed with mild hemophilia $\mathrm{A}$ in his teens after he exhibited difficulty achieving hemostasis after traumatic injury, which resolved without any treatment. The FVIII:C was 5-10\%. There was no history of hemophilia in his family. He had no bleeding episodes requiring FVIII replacement therapy until the age of 64. At the age of 65, he was given plasma-derived FVIII (pdFVIII) products (30 U/kg, every 12 hours over 3 days) for the first time because of bleeding at the right elbow. At the age of 68 , he was given pdFVIII for the second time because of intraperitoneal bleeding. He presented to the emergency room with hematemesis and abdominal pain. Laboratory examinations revealed a low level of hemoglobin $(9.8 \mathrm{~g} / \mathrm{dL})$. Abdominal computed tomography showed intraperitoneal bleeding. Emergency selective angiography revealed bleeding from the gastroepiploic artery. Trans-arterial embolization was successfully performed. He was given pdFVIII (30 U/kg, every 12 hours) for 10 days. The FVIII:C increased following the infusion of pdFVIII as expected, and an inhibitor detection test (Bethesda assay) was negative.

\footnotetext{
${ }^{1}$ Departments of Hematology, Clinical Immunology and Infectious Diseases, Ehime University Graduate School of Medicine, Japan, ${ }^{2}$ Department of Internal Medicine, Uwajima City Hospital, Japan and ${ }^{3}$ Division of Blood Transfusion and Cell Therapy, Ehime University Graduate School of Medicine, Japan

Received: March 7, 2018; Accepted: March 29, 2018; Advance Publication by J-STAGE: June 6, 2018

Correspondence to Dr. Jun Yamanouchi, yamanouc@m.ehime-u.ac.jp
} 


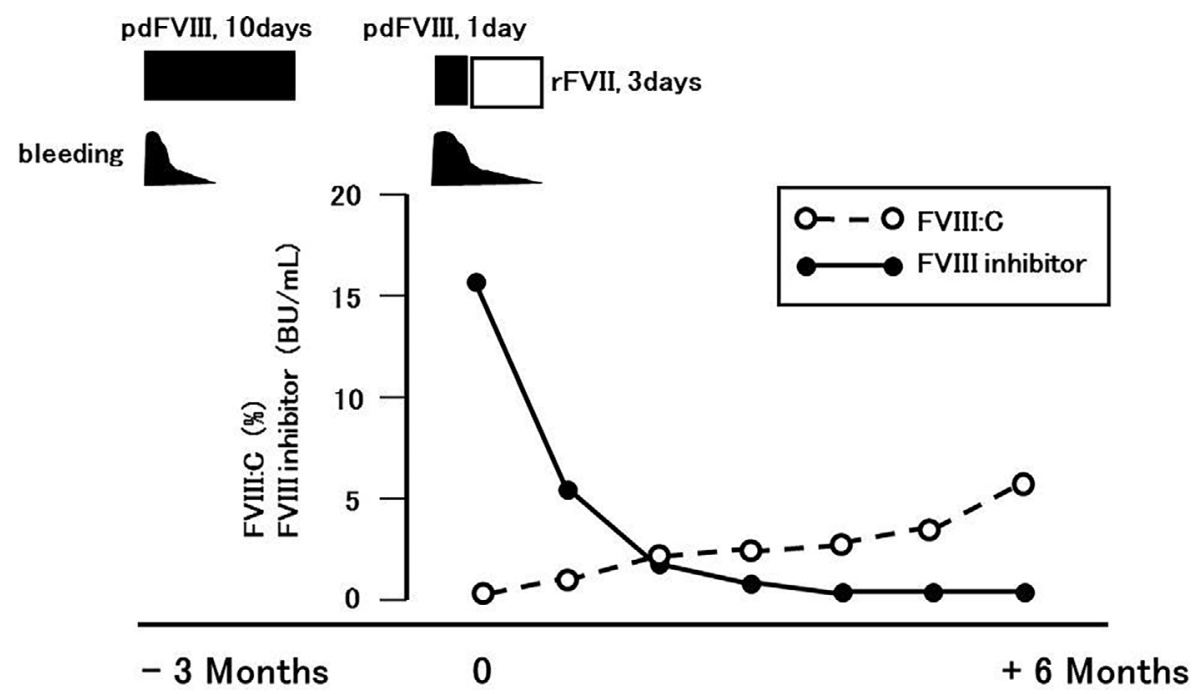

Figure 1. Clinical course.

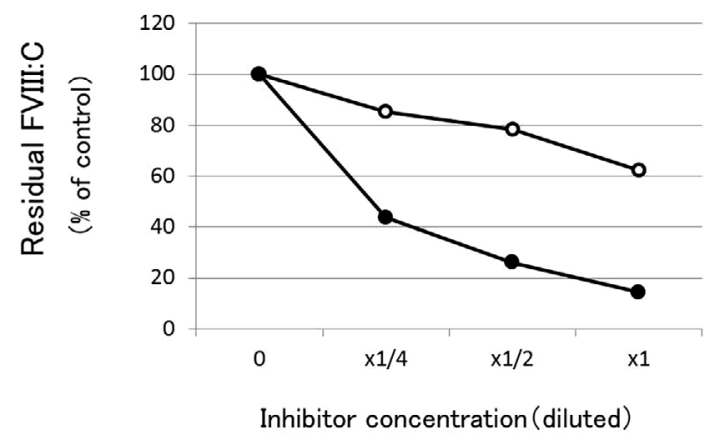

Figure 2. Differing effects of the patient's FVIII inhibitor on exogenous wild-type FVIII and autologous mutant FVIII. The patient's plasma containing the inhibitor was treated at $56^{\circ} \mathrm{C}$ for $30 \mathrm{~min}$ in order to eliminate residual VIII:C. Treated plasma (inhibitor concentration: $\times 1$ ) was diluted 2 - and 4 -fold with HEPES buffer and incubated with normal pooled plasma (closed circle), the patient's plasma stored before inhibitor development (open circle), or buffer (inhibitor concentration: 0) at $37^{\circ} \mathrm{C}$ for $2 \mathrm{~h}$. The residual VIII:C was measured using a onestage clotting assay and expressed as a percentage of the value for each plasma sample incubated with buffer.

Three months later, hemorrhaging occurred at the bilateral femoral muscle and pharynx. He was given pdFVIII (30 U/ $\mathrm{kg}$, every 12 hours) twice but had difficulty achieving hemostasis. We immediately measured the FVIII:C after the administration of pdFVIII and found that it was $<1 \%$. Bethesda assay of his FVIII inhibitor showed a value of 15.5 $\mathrm{BU} / \mathrm{mL}$. He was administered a recombinant FVIIa product (Novo-Seven; Novo Nordisk, Tokyo, Japan), which stopped the hemorrhaging. There were no bleeding episodes that required FVIII replacement therapy. The titer of the inhibitor spontaneously decreased and disappeared six months later. Now, his FVIII:C is 5\% (Fig. 1).

We examined whether or not the patient's inhibitor neutralized endogenous mutant FVIII. The patient's plasma con- taining the FVIII inhibitor was treated at $56^{\circ} \mathrm{C}$ for 30 minutes to eliminate residual endogenous FVIII:C. A mixture of treated plasma and the patient's plasma in which the inhibitor had disappeared was subjected to the Bethesda assay. The patient's inhibitor exhibited a linear dose-response relationship characteristic for type I inhibition kinetics when his plasma was mixed with normal pooled plasma. Unfortunately, we were unable to collect his plasma on admission, so we used plasma collected seven days later. The FVIII:C was $1.4 \%$, and the FVIII inhibitor was $4.8 \mathrm{BU} / \mathrm{mL}$. The patient's inhibitor in treated plasma neutralized mutant FVIII:C in the patient's plasma without the inhibitor by approximately $40 \%$ and neutralized wild-type FVIII:C in normal pooled plasma completely (Fig. 2).

A venous blood sample was collected from the patient, and genomic DNA was extracted from leukocytes according to the standard procedure using the QIAamp DNA Blood Mini kit (QIAGEN, Hilden, Germany). The entire FVIII gene-coding regions and exon/intron boundaries were amplified by polymerase chain reaction (PCR) with appropriate primers. The PCR products were purified and directly sequenced using a 3500 Genetic Analyzer (Applied Biosystems, Tokyo, Japan).

This study was approved by the Ethics Committee of Ehime University Graduate School of Medicine. The subject gave his written informed consent. A sequence analysis of FVIII in this patient showed a 1784T $>$ G alteration (p.Phe 595Cys) in exon 12 of the FVIII gene.

\section{Discussion}

We encountered a patient with mild hemophilia who carried the Phe595Cys mutation in FVIII and developed an inhibitor. According to the Factor VIII Gene (F8) Variant Database (http://www.factorviii-db.org/), this is the first report in which the Phe595Cys mutation was found to be causative for mild hemophilia and associated with inhibitor develop- 
ment; however, the Phe595Ser mutation was previously reported to be causative for severe hemophilia. In addition, this study showed that the inhibitor recognized exogenous wild-type FVIII and autologous mutant FVIII. Previous studies reported that certain mutations in the FVIII gene in mild hemophilia are associated with a greater risk of inhibitor development. Missense mutations introducing a cysteine residue may alter disulphide bridge formation, leading to a gross aberrant conformation. Certain mutations, including Arg593Cys and Trp2229Cys (4, 5), have been associated with inhibitor development. Among them, Arg593Cys was found to have a 10-fold higher risk of developing inhibitors (5). The Phe595Cys mutation in this study may also induce the development of inhibitors by the same mechanism. In the present study, we expanded the list of genetic mutations associated with the FVIII inhibitor to include Phe595 Cys.

In addition to genetic risk factors, some environmental factors are associated with inhibitor development in mild hemophilia, including intensive FVIII treatments, old age at the first exposure, and a high number of peak treatments (3). Our patient was subjected to intensive FVIII treatments twice, and the inhibitor developed after the second intensive therapy for intraperitoneal bleeding. The cause of his intraperitoneal bleeding was unclear. The risk of inhibitor development after surgery has been demonstrated in patients with severe and mild hemophilia $(5,6)$. FVIII infused into patients with hemophilia is recognized as being different from autologous FVIII, generating an alloantibody. Therefore, we believe that the inhibitor that developed in our patient was directed against the A2 domain, in which the mutated residue (Phe595) was located. Previous studies have shown that the epitopes of almost all FVIII inhibitors were located in the A2 and/or A3-C1-C2 domains, suggesting the high immunogenicity of these domains (7). The Phe595Cys mutation may induce conformational changes in the A2 domain of endogenous mutant FVIII, resulting in the development of an inhibitor against the A2 domain of exogenous wildtype FVIII. Most inhibitors in patients with mild hemophilia have been reported to cross-react with autologous FVIII, resulting in little or no residual FVIII:C $(4,5)$. However, we previously reported that a mild hemophilia patient with the Glu272Lys mutation in FVIII developed an exogenous FVIII-specific inhibitor (8).

Viel et al. reported that the prevalence of six wild-type FVIII proteins (the H1-H6 haplotypes) was dependent on ethnicity (9). They speculated that a mismatch in the FVIII haplotype between the FVIII concentrate and its recipients, particularly in immunodominant epitopes, led to an increase in the frequency of inhibitor development. They focused on four different amino acid polymorphisms (R484H, R776G, D1241E, and M2238V), based on a non-synonymous single nucleotide polymorphism. We tested our patient for these polymorphisms, and the results revealed that he had the $\mathrm{H} 1$ haplotype, which is the most frequent haplotype found in Japanese people (10).
Haplotypes of the immune response factor have been investigated in relation to the risk of inhibitor development. Pavlova et al. reported that the incidence of inhibitor development was related to different TNF- $\alpha /$ IL-10 genotype combinations. A strong association was observed between the high-TNF- $\alpha$ and high-IL-10 haplotypes [TNF- $\alpha-308$ (AA/ GA) and IL-10-1082 (GG)] and inhibitor formation (OR 4.4) (11). TNF- $\alpha$ and IL-10 analyses (TNF- $\alpha-308 \mathrm{G} / \mathrm{G}$ and IL-10-1082G/A) revealed that our patient was at low risk of developing inhibitors.

Whether or not a specific mutation is associated with the development of an inhibitor that does not cross-react with autologous mutant FVIII is unclear at present. DDAVP may be an effective therapeutic option for patients with a noncross reactive inhibitor but may be ineffective for patients with a cross-reactive inhibitor.

In conclusion, we encountered a mild hemophilia patient with the Phe595Cys mutation in FVIII who developed an exogenous FVIII inhibitor. More data on the link between the genotypes and characteristics of inhibitors in mild hemophilia are needed. This information will facilitate more appropriate patient management and help better define the molecular mechanisms underlying inhibitor development in mild hemophilia.

The authors state that they have no Conflict of Interest (COI).

\section{References}

1. Castaman G, Eckhardt C, van Velzen A, et al. Emerging issures in diagnosis, biology, and inhibitor risk in mild hemophilia A. Semin Thromb Hemost 42: 507-512, 2016.

2. Eckhardt CL, van Velzen AS, Peters M, et al. Factor VIII gene (F8) mutation and risk of inhibitor development in nonsevere hemophilia A. Blood 122: 1954-1962, 2013.

3. Mauser-Bunschoten EP, Den Uijl IE, Schutgens RE, et al. Risk of inhibitor development in mild haemophilia A increases with age. Haemophilia 18: 263-267, 2012.

4. Hay CR, Ludlam CA, Colvin BT, et al. Factor VIII inhibitors in mild and moderateseverity haemophilia A. Thromb Haemost 79: 762-766, 1998.

5. Eckhardt CL, Menke LA, Van Ommen $\mathrm{CH}$, et al. Intensive perioperative use of factor VIII and the Arg593>Cys mutation are risk factors for inhibitor development in mild/moderate hemophilia A. J Thromb Haemost 7: 930-937, 2009.

6. Eckhardt CL, Mauser-Bunschoten EP, Peters M, et al. Inhibitor incidence after intensive FVIII replacement for surgery in mild and moderate haemophilia A: a prospective national study in the Netherlands. Br J Haematol 157: 747-752, 2012.

7. Shima M. Characterization of factor VIII inhibitors. Int J Hematol 83: 109-118, 2006.

8. Yamanouchi J, Hato T, Niiya T, et al. Development of exogenous FVIII-specific inhibitor in a mild haemophilia patient with Glu272 Lys mutation. Haemophilia 20: e171-e182, 2014.

9. Viel KR, Ameri A, Abshire TC, et al. Inhibitors of factor VIII in black patients with hemophilia. N Engl J Med 360: 1618-1627, 2009.

10. Inaba H, Shinozawa K, Hagiwara T, et al. Factor VIII haplotypes of Japanese population show similarity to those of Caucasian populations. Haemophilia 18: e43-e59, 2012.

11. Pavlova A, Delev D, Lacroix-Desmazes S, et al. Impact of poly- 
morphisms of the major histocompatibility complex class II, interleukin-10, tumor necrosis factor- $\alpha$ and cytotoxic Tlymphocyte antigen-4 genes on inhibitor development in severe hemophilia A. J Thromb Haemost 7: 2006-2015, 2009.
The Internal Medicine is an Open Access journal distributed under the Creative Commons Attribution-NonCommercial-NoDerivatives 4.0 International License. To view the details of this license, please visit (https://creativecommons.org/licenses/ by-nc-nd/4.0/).

(C) 2018 The Japanese Society of Internal Medicine Intern Med 57: 3179-3182, 2018 